\title{
Effect of synchronous solitary bone metastasectomy and lung cancer resection on non-small cell lung cancer patients
}

\author{
TIANCHENG ZHAO, ZONGLI GAO, WEIMING WU, WEIWEI HE and YI YANG \\ Department of Cardiothoracic Surgery, Shanghai Sixth People's Hospital, Shanghai 200233, P.R. China
}

Received November 17, 2014; Accepted October 9, 2015

DOI: $10.3892 / \mathrm{ol} .2016 .4190$

\begin{abstract}
Lung cancer is the most frequent cause of cancer-associated mortality among men and women globally. The skeleton is one of the most common metastatic sites. The majority of patients exhibiting bone metastases are treated using systemic therapy or symptom-based palliative approaches without surgery. The present study attempted to improve the therapeutic effects of synchronous surgeries in resectable non-small cell lung cancer patients exhibiting solitary bone metastasis. A total of 5 patients underwent synchronous lung cancer resections and solitary bone metastasectomies between October 2009 and November 2011 in the Department of Cardiothoracic Surgery, Shanghai Sixth People's Hospital (Shanghai, China). All patients had received fluorodeoxyglucose positron emission tomography-computed tomography or bone scintigraphy to demonstrate the presence of solitary bone metastasis and to exclude the presence of metastases at alternative sites. The patients received standard lung cancer and mediastinal lymph node resections. In addition, bone lesions were assessed by orthopedists and operated on synchronously with standard procedures. Following surgery, all patients were administered standard chemotherapeutic regimens. Perioperative indicators, including time for thoracic drainage, length of hospital stay, incidence of post-operative complications and progression-free survival (PFS) time, were observed. The average time for post-operative drainage was $4.6 \pm 1.1$ days, and the average length of post-operative hospitalization was $8.8 \pm 2.2$ days. All procedures were performed safely with no serious complications. The PFS of the patients was $13.2 \pm 7.7$ months. While 2 patients presenting with spinal metastases succumbed at $\sim 1$ year post-surgery, the remaining 3 patients presenting with limb bone metastases survived for $>16$ months post-surgery, and were alive at the
\end{abstract}

Correspondence to: Dr Yi Yang, Department of Cardiothoracic Surgery, Shanghai Sixth People's Hospital, 600 Yishan Road, Shanghai 200233, P.R. China

E-mail: yiyangqwe345@163.com

Key words: solitary bone metastasis, metastasectomy, non-small cell lung cancer, synchronous surgery last follow-up. In conclusion, the present study indicated that a synchronous metastasectomy and lung tumor resection is a safe method of treatment. The PFS time and survival results demonstrated that on the rare occasion that a patient exhibits solitary bone metastasis, aggressive surgical treatment may be a potential therapeutic option.

\section{Introduction}

Lung cancer is the most frequent cause of cancer-associated mortality among men and women globally (1). The skeletal system is one of the most common sites of metastasis. A large number of lung cancer patients do not have the option to undergo surgery at the time of initial diagnosis due to the presence of metastases. In total, 30-40\% of patients exhibiting advanced lung cancer go on to develop skeletal metastases, which may cause skeletal-related events (SREs), including spinal cord compression, severe bone pain, pathological fractures and potentially life-threatening hypercalcemia of malignancy, requiring treatment with consecutive orthopedic surgery or palliative radiotherapy $(2,3)$. In general, the majority of these patients are treated using systemic therapy or a symptom-based palliative approach. Overall, median survival rates are poor, ranging between 4 and 11 months (4). However, $7 \%$ of patients exhibiting metastatic non-small cell lung cancer (NSCLC) will present with solitary metastasis following a complete evaluation (5).

According to the guidelines for NSCLC published by the National Comprehensive Cancer Network (6), the therapeutic strategies for solitary brain or adrenal metastasis are easily identified. Synchronous brain or adrenal metastasectomy is the recommended treatment if the tumor in the lung can be totally resected (7-9). However, we could not identify any suggestions for the appropriate synchronous treatment of solitary bone metastasis and NSCLC. A limited number of case reports concerning a synchronous bone metastasectomy and lung tumor resection were identified in Japanese studies. In these studies, patients achieved long-term survival following synchronous bone metastasectomies and lung tumor resections (10-12). Bae et al (13) demonstrated that although solitary bone metastasis occurred much less frequently than metastasis at two or more sites, it was a predictor of a good prognosis.

The present study attempted to assess the safety and effectiveness of a synchronous complete lung cancer resection and 
Table I. General clinical characteristics of patients.

\begin{tabular}{|c|c|c|c|c|c|c|c|c|}
\hline \multirow[b]{2}{*}{ Patient no. } & \multicolumn{2}{|c|}{ Disease location } & \multicolumn{2}{|c|}{ Type of resection } & \multirow[b]{2}{*}{ Pathology } & \multirow{2}{*}{$\begin{array}{l}\text { Lymph node } \\
\text { positivity }\end{array}$} & \multirow{2}{*}{$\begin{array}{l}\text { PFS, } \\
\text { months }\end{array}$} & \multirow{2}{*}{$\begin{array}{c}\text { Survival time, } \\
\text { months }\end{array}$} \\
\hline & Primary & Metastasis & Primary & Metastasis & & & & \\
\hline 1 & RUL & R scapula & VATS lobectomy & $\mathrm{BR}$ & Adenocarcinoma & No & 26 & $35+^{\mathrm{a}}$ \\
\hline 2 & ML & T9 spine & Lobectomy & $\mathrm{TR}+\mathrm{IF}$ & Adenocarcinoma & No & 6 & 8 \\
\hline 3 & ML & $\mathrm{T} 4$ spine & Lobectomy & $\mathrm{TR}+\mathrm{IF}$ & $\mathrm{LCC}$ & No & 10 & 12 \\
\hline 4 & RUL & L humerus & VATS lobectomy & $\mathrm{TR}+\mathrm{PR}$ & Adenocarcinoma & No & 14 & $14+^{\mathrm{a}}$ \\
\hline 5 & ML & $\mathrm{R}$ femur & VATS lobectomy & $\mathrm{TR}+\mathrm{PR}$ & Adenocarcinoma & No & 10 & $10+^{\mathrm{a}}$ \\
\hline
\end{tabular}

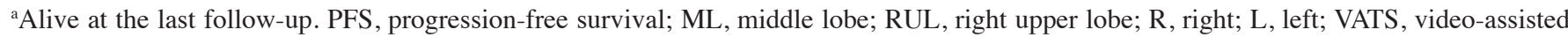
thoracoscopic surgery; BR, bone resection; TR, tumor resection; IF, internal fixation; PR, prosthetic replacement; LCC, large cell carcinoma.
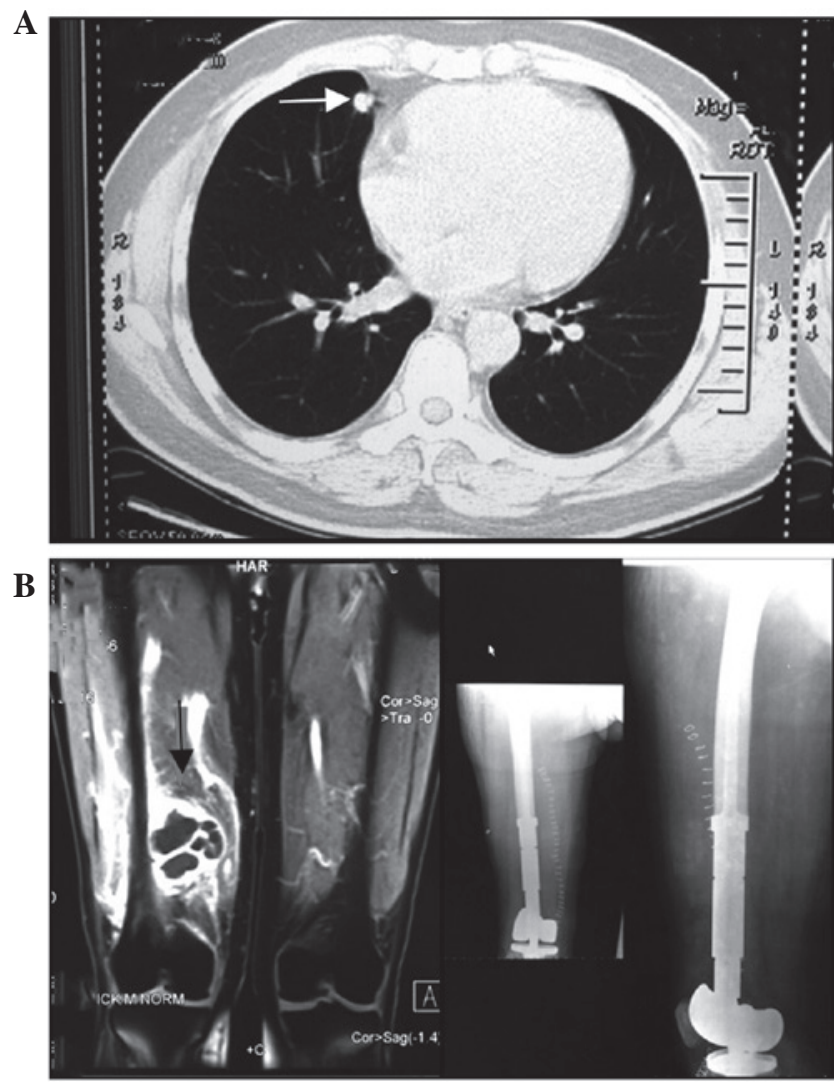

Figure 1. Images captured following metastasectomy in a 47-year-old male patient presenting with a (A) middle lobe nodule and (B) right femur metastasis. White arrow indicates a right middle lobe nodule. Black arrow indicates right femur metastasis.

solitary bone metastasectomy. Perioperative indicators and progression-free survival (PFS) were analyzed. In addition, post-operative survival time was observed.

\section{Materials and methods}

Patient clinical characteristics. A total of 5 NSCLC patients exhibiting solitary bone metastasis were enrolled into the present study between October 2009 and November 2011. Prior to surgery, patients underwent a series of standard clinical examinations, including fluorodeoxyglucose-positron emission tomography-computed tomography $\left({ }^{18} \mathrm{FDG}\right.$-PET-CT) or bone scintigraphy and brain magnetic resonance imaging, in order to demonstrate the presence of solitary bone metastasis and to exclude the presence of metastasis in other organs. The study was approved by the ethics committee of Shanghai Sixth People's Hospital (Shanghai, China) and written informed consent was obtained from all patients.

Treatment planning. Bone metastases were assessed using PET-CT and isotope bone scans by orthopedists and identified as being resectable. Lung tumors were additionally observed to be completely resectable. Metastases/tumors were considered resectable if they were isolated, without invasion of the vital organs, and could be removed completely with wide resection margins. All patients were informed of the risks and significance of synchronous surgeries and accepted the therapeutic schedule. For primary lung tumors, thoracotomy or video-assisted thoracoscopic surgery (VATS) lobectomies and systematic mediastinal lymph node resections were performed. For bone metastases, limb metastases were resected and reconstructed using prosthetic replacement. Spine metastases were resected and also underwent an internal fixation or interventional embolization.

Following tumor resection, all patients were administered four 4-week cycles of standard first-line chemotherapy (75 mg/m² docetaxel, day $1 ; 75 \mathrm{mg} / 2$ cisplatin, day 1) and bisphosphonate (4 mg Zometa) treatment. Perioperative indicators, including time of thoracic drainage, incidence of post-operative complications, length of hospital stay and PFS, were observed. Data is expressed as the median \pm standard deviation.

\section{Results}

Patient clinical characteristics. Table I summarizes the detailed clinical characteristics of each patient enrolled in the present study. The histologies of the lung cancer cases included 4 patients with adenocarcinoma and 1 patient with large cell carcinoma. A total of 2 patients had lung masses located in the right upper lobe and 3 had lung masses located in the middle lobe. In addition, 2 patients possessed metastasis to the spine and exhibited an SRE of spinal cord compression. The remaining 3 patients demonstrated no SREs, 
Table II. Surgical features of patients $(n=5)$.

\begin{tabular}{lc}
\hline Surgical feature & Value \\
\hline Median age (range), years & $54.6(47-72)$ \\
Gender & \\
Male & 4 \\
Female & 1 \\
Thoracic drainage, days & $4.6 \pm 1.1$ \\
Post-operative hospital stay, days & $8.8 \pm 2.2$ \\
PFS, months & $12.8 \pm 7.7$ \\
Post-operative survival, months & $15.8 \pm 10.9$ \\
\hline
\end{tabular}

PFS, progression-free survival. Data are expressed as median \pm standard deviation.
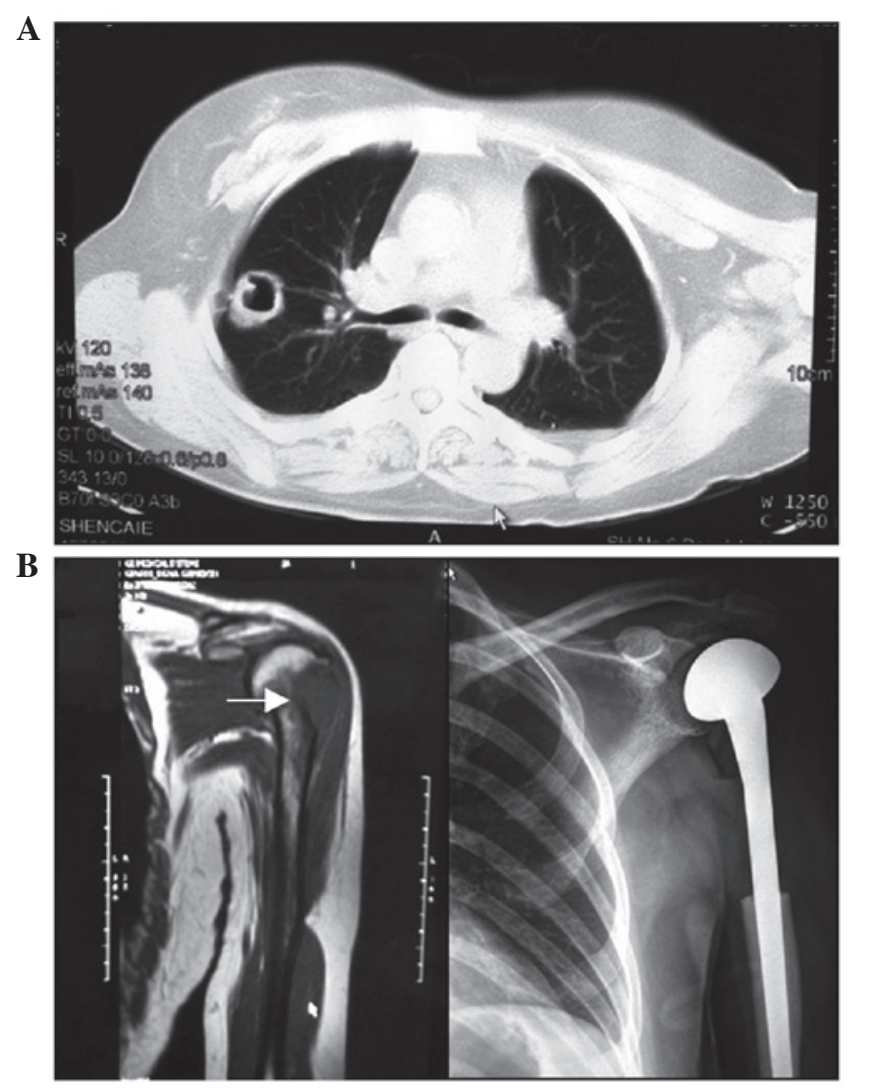

Figure 2. Images captured following metastasectomy in a 47-year-old woman presenting with a (A) right upper lobe mass and (B) left humerus lesion. White arrow indicates left humerus metastasis.

however, exhibited a solitary metastasis to the right scapula, left humerus and right femur, respectively. In total, 2 patients underwent thoracotomy procedures, and 3 underwent VATS. Systematic mediastinal lymph node resection is essential for lobectomy, however, lymph node positivity was not identified in the patients in the present study.

Imaging results. Fig. 1 shows images captured following metastasectomy in a 47 -year-old male patient presenting with a middle lobe nodule and right femur metastasis. The patient underwent a synchronous lobectomy and metastasectomy, and reconstruction with a prosthetic replacement in April 2011. A total of two lesions identified in the patient were pathologically proven to be adenocarcinoma. Additional distant bone metastases were identified in the patient 10 months after the initial surgery, therefore further chemotherapy $\left(75 \mathrm{mg} / \mathrm{m}^{2}\right.$ docetaxel, day $1 ; 75 \mathrm{mg} / 2$ cisplatin, day 1 ; four 4 -week cycles) was administered and the patient remains under follow-up.

Fig. 2 shows images captured following metastasectomy in a 47-year-old woman presenting with a right upper lobe mass and left humeral lesion. The patient underwent a synchronous surgery and reconstruction with prosthetic replacement in September 2011. The lesions identified in the patient were pathologically proven to be adenocarcinoma. For cancers that begin in glandular secretory cells, a well-differentiated low grade tumor resembles the normal glandular structure. Poorly-differentiated high grade adenocarcinomas do not resemble normal glands and may be detected by positive staining for mucin, which the glands produce. Adenocarcinoma may also be distinguished by positive staining for transcription termination factor, RNA polymerase I, a cell marker for adenocarcinoma. In the present study, the cancer cells formed matured tubular, acinus structure or columnar cells lining the papillary structure, or the lesions exhibited mucus secretion. Diagnosis of the lesions was based on the observation that the cancerous tissues possessed characteristics of adenoid differentiation. The patient survived for 14 months with no evidence of recurrence and remains under follow-up.

Fig. 3 shows images captured from a 72-year-old man presenting with a right upper lobe mass and right scapula metastasis. It was difficult to identify metastasis using X-ray imaging, however, these difficulties were simplified by using ${ }^{18}$ FDG-PET-CT scanning. The patient underwent a VATS lobectomy and right scapula resection in September 2009, and had a PFS time of 26 months. The patient remains alive following the administration of multimodal therapy with thymosin (4 mg, twice a week) for 3 months and Traditional Chinese Medicine for 6 months. ${ }^{18}$ FDG-PET-CT demonstrates increased diagnostic value (sensitivity and specificity) for the diagnosis of bone metastasis from lung cancer compared with alternative imaging methods (14).

Surgical outcomes. Additional perioperative data is summarized in Table II. The median patient age was 54.6 years, with a range of 47-72 years. All procedures were performed safely, and without the occurrence of serious complications. The median thoracic drainage time was $4.6 \pm 1.1$ days, and the length of post-operative hospitalization was $8.8 \pm 2.2$ days. The PFS time of the patients was $13.2 \pm 7.7$ months. All patients were administered standard first-line chemotherapy and bisphosphonate treatment following surgery. The 2 patients who initially exhibited spinal metastasis, demonstrated malignant pericardial and thoracic effusion without paralysis $\sim 1$ year subsequent to the initial surgery and rapidly succumbed to the disease. The remaining 3 patients exhibiting limb bone metastases experienced an increased average PFS time of $\sim 16$ months. These patients have survived for $>2$ years following the initial surgery and remain under follow-up. The post-operative survival time of all patients was $15.8 \pm 10.9$ months. 


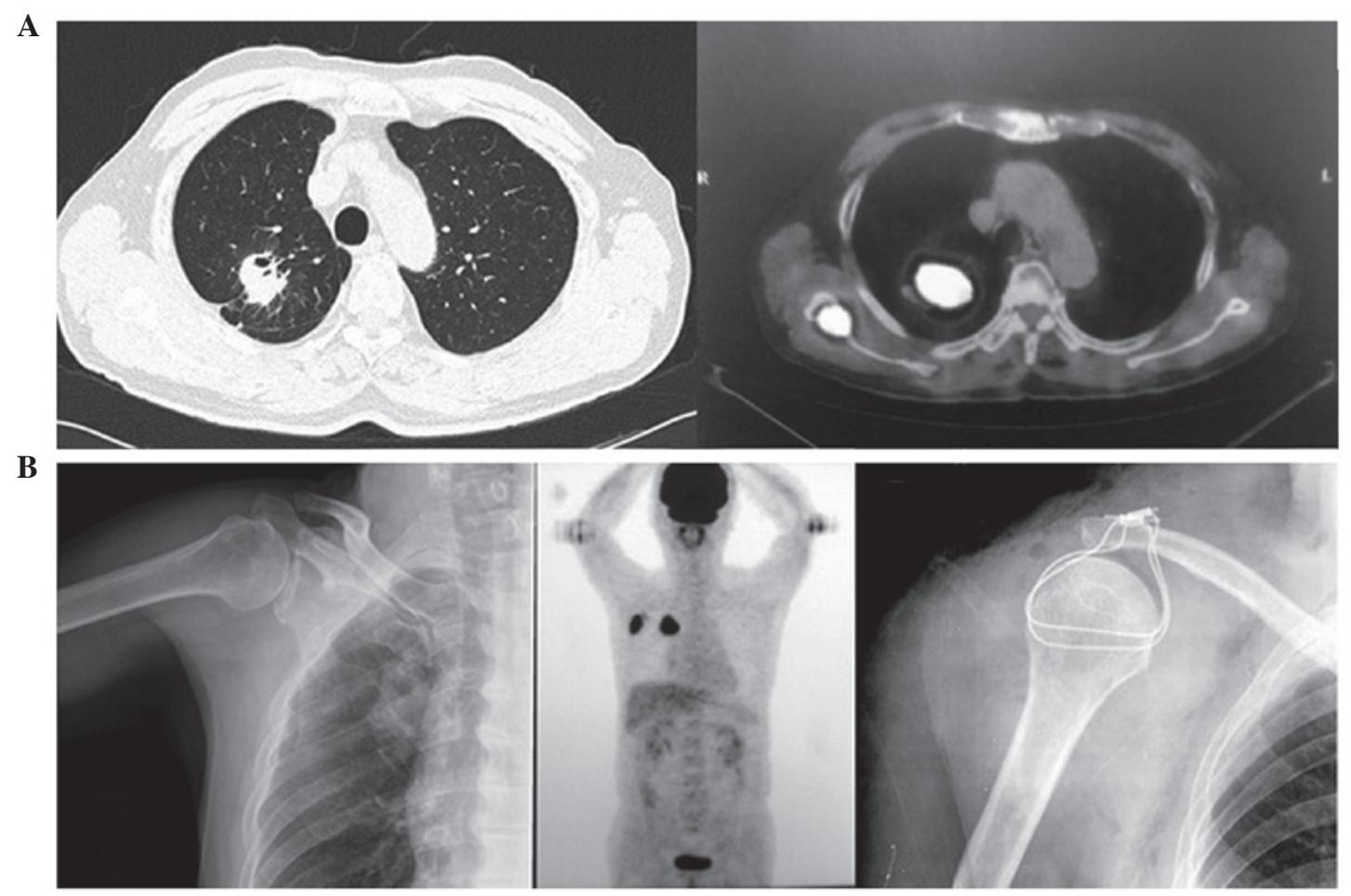

Figure 3. Images captured in a 72-year-old man presenting with a (A) right upper lobe mass and (B) right scapula metastasis.

\section{Discussion}

Following more than a decade of investigation, the treatment of synchronous single-organ metastatic NSCLC remains under debate. Considering the low incidence of this particular presentation of NSCLC, it remains unlikely that large prospective randomized studies will be conducted. Therefore, knowledge has to be constructed from the results of retrospective studies (5). Bone metastasis identified at the initial diagnosis of NSCLC typically indicates the possibility of consequent tumor spread and a poor patient prognosis (15). Decroisette et al (16) reported a poor prognosis among NSCLC patients exhibiting bone metastatic disease. In the study, the median survival time was observed to be 5.8 months, and the 1- and 2-year survival rates were 22 and $7 \%$, respectively. There was no significant difference observed in overall survival between the patients with and without SRE at enrollment (16). In a study performed by Sugiura et al (17), the cumulative survival rate following bone metastasis was observed to be $59.9 \%$ for 6-month survival, $31.6 \%$ for 1 -year survival and $11.3 \%$ for 2 -year survival. The mean survival time was 9.7 months (17).

Among the patients in the present study, a PFS time of 13.2 \pm 7.7 months was obtained. This meant that patients remained tumor-free for $>1$ year after surgery. We propose that it is important to prolong the overall patient survival time, and that this has a positive effect on patient psychology. In addition, the average post-operative survival time of $15.8 \pm 10.9$ months in the present study was an improvement on the times in aforementioned studies. In a study performed by Weiss and Wedin (18), patients exhibiting spinal metastases possessed a significantly poorer prognosis in a patient cohort with skeletal metastasis. However, 16/31 patients exhibiting spinal metastases demonstrated a considerable improvement in neurological function following surgery. In the present study, the 2 patients exhibiting spinal metastases possessed reduced PFS and post-operative survival times, however, demonstrated no paralysis or neurological symptoms prior to mortality. In the present study, the 2 patients exhibiting extremity metastases complained of pain prior to surgery, which was subsequently relieved, without loss of limb function. Orthopedic surgeries may reserve the majority of function of the extremities and reduce pain, decreasing the rate of disability and pathological fracture, and improving patient quality of life (19).

There is currently a general consensus concerning synchronous metastasectomy of solitary adrenal or brain metastasis in NSCLC. We propose that solitary bone metastasis should be considered to have a surgically treatable status. Due to the low occurrence rate of solitary bone metastasis in NSCLC patients, the sample investigated in the present study was small, and it was difficult to achieve a definitive conclusion. However, the present study demonstrated an acceptable PFS time, and overall survival results were more positive compared with those achieved with conservative therapy. In conclusion, an aggressive regimen of synchronous metastasectomy and primary tumor resection may be beneficial for selected patients, leading to prolonged survival times and an improved quality of life.

\section{Acknowledgements}

The abstract of the present study was previously published in the proceedings of the 15th World Conference on Lung Cancer, Oct 27-31, 2013, in Sydney, and was published as abstract PL03.01 in J Thorac Oncol 8 (Suppl 2) 2013.

\section{References}

1. Jemal A, Bray F, Center MM, Ferlay J, Ward E and Forman D: Global cancer statistics. CA Cancer J Clin 61: 69-90, 2011. 
2. Rosen LS, Gordon D, Tchekmedyian NS, Yanagihara R, Hirsh V, Krzakowski M, Pawlicki M, De Souza P, Zheng M, Urbanowitz G, et al: Long-term efficacy and safety of zoledronic acid in the treatment of skeletal metastases in patients with nonsmall cell lung carcinoma and other solid tumors: A randomized, Phase III, double-blind, placebo-controlled trial. Cancer 100: 2613-2621, 2004.

3. Price N: Bisphosphonates to prevent skeletal morbidity in patients with lung cancer with bone metastases. Clin Lung Cancer 5: 267-269, 2004.

4. Groome PA, Bolejack V, Crowley JJ, Kennedy C, Krasnik M, Sobin LH and Goldstraw P; IASLC International Staging Committee; Cancer Research and Biostatistics; Observers to the Committee; Participating Institutions: The IASLC Lung Cancer Staging Project: Validation of the proposals for revision of the $\mathrm{T}, \mathrm{N}$, and $\mathrm{M}$ descriptors and consequent stage groupings in the forthcoming (seventh) edition of the TNM classification of malignant tumours. J Thorac Oncol 2: 694-705, 2007.

5. Pfannschmidt J and Dienemann H: Surgical treatment of oligometastatic non-small cell lung cancer. Lung Cancer 69: 251-258, 2010.

6. Ettinger DS, Wood DE, Akerley W, Bazhenova LA, Borghaei H, Camidge DR, Cheney RT, Chirieac LR, D'Amico TA, Demmy TL, et al: Non-small cell lung cancer, version 6.2015. J Natl Compr Canc Netw 13: 515-524, 2015.

7. Hu C, Chang EL, Hassenbusch SJ III, Allen PK, Woo SY, Mahajan A, Komaki R and Liao Z: Nonsmall cell lung cancer presenting with synchronous solitary brain metastasis. Cancer 106: 1998-2004, 2006.

8. Raviv G, Klein E, Yellin A, Schneebaum S and Ben-Ari G: Surgical treatment of solitary adrenal metastases from lung carcinoma. J Surg Oncol 43: 123-124, 1990.

9. Reyes L, Parvez Z, Nemoto T, Regal AM and Takita H: Adrenalectomy for adrenal metastasis from lung carcinoma. J Surg Oncol 44: 32-34, 1990.
10. Agarwala AK and Hanna NH: Long-term survival in a patient with stage IV non-small-cell lung carcinoma after bone metastasectomy. Clin Lung Cancer 6: 367-368, 2005

11. Hirano Y, Oda M, Tsunezuka Y, Ishikawa N and Watanabe G Long-term survival cases of lung cancer presented as solitary bone metastasis. Ann Thorac Cardiovasc Surg 11: 401-404, 2005.

12. Ono K, Nagashima A, Yokoyama E, Nose N and Yasumoto K: Long-term survival after surgical resection of bone metastasis from lung cancer. Kyobu Geka 63: 216-219, 2010 (In Japanese).

13. Bae HM, Lee SH, Kim TM, Kim DW, Yang SC, Wu HG, Kim YW and Heo DS: Prognostic factors for non-small cell lung cancer with bone metastasis at the time of diagnosis. Lung Cancer 77: 572-577, 2012.

14. Qu X, Huang X, Yan W, Wu L and Dai K: A meta-analysis of ${ }^{18} \mathrm{FDG}-\mathrm{PET}-\mathrm{CT},{ }^{18} \mathrm{FDG}-\mathrm{PET}, \mathrm{MRI}$ and bone scintigraphy for diagnosis of bone metastases in patients with lung cancer. Eur J Radiol 81: 1007-1015, 2012.

15. Lopes G, Piedade A, Goes L, Alves M, and Balu S: Diagnoses and treatment patterns for Non-small cell lung cancer (Nsclc) within the private health system in Brazil. Value Health 18: A825, 2015.

16. Decroisette C, Monnet I, Berard H, Quere G, Le Caer H, Bota S, Audigier-Valette C, Geriniere L, Vernejoux JM and Chouaid C; Groupe Français de Pneumo-Cancérologie 0601 Team: Epidemiology and treatment costs of bone metastases from lung cancer: A French prospective, observational, multicenter study (GFPC 0601). J Thorac Oncol 6: 576-582, 2011.

17. Sugiura H, Yamada K, Sugiura T, Hida T and Mitsudomi T: Predictors of survival in patients with bone metastasis of lung cancer. Clin Orthop Relat Res 466: 729-736, 2008.

18. Weiss RJ and Wedin R: Surgery for skeletal metastases in lung cancer. Acta Orthop 82: 96-101, 2011.

19. Bao DM, Li N and Xia L: Risk assessment and decision-making for patients undergoing orthopedic surgery. J Orthop Surg Res 10: 169, 2015. 